\title{
Weighted Residual Approach for Bending Analysis of Nanobeam Using by Modified Couple Stress Theory
}

\author{
Mustafa Özgür Yaylı ${ }^{a^{*}}$, Togay Küpeli ${ }^{b}$, Yakup Harun Çavuş $^{c}$ \\ ${ }^{a, b, c}$ Department of Civil Engineering, Uludă̆ University, Bursa, Turkey

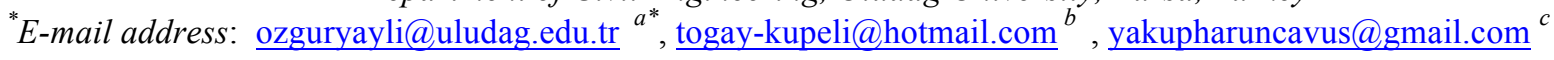 \\ ORCID numbers of authors: \\ 0000-0003-2231-170X ${ }^{a_{*}}, 0000-0002-5921-8667^{b}, 0000-0002-6607-9650^{c}$
}

Received date: 04.05.2021

Accepted date: 18.06.2021

\begin{abstract}
With the development of nanotechnology, interest in nanomaterials has increased significantly in recent years. This study examines the bending analysis of a nanobeam with modified couple stress theory and weighted residual methods. The formulas derived for calculating bending analysis results in the article has been found by using Weighted Residual Method. The results have compared to show effects on nanobeam and the calculated values are shown in the graphs and tables. The results which is obtained in the article are compared with the results already found in the literature and it was observed that they are consistent.
\end{abstract}

Keywords: Nanotechnology, Bending, Nanobeam, Modified Couple Stress Theory, Weighted Residual Methods.

\section{Introduction}

Recently, the use of nanomaterials has been very wide with a high interest. For instance; nanorods are used in electronics, material and manufacturing industry, medical and healthcare, aviation and space research, environment and energy, defense sector, biomedical industries and various engineering structures. Nanotechnology is an evolving technology, and we can see more applications in nanotechnology. Nowadays, it is clear that it will spread much wider in future. Nanotechnology can be said to be a golden key for technology, as technology developments continue and the for human needs is now being ensured by smaller materials and tools.

It is anticipated that nanotechnology will benefit humanity in all areas as a result of its correct and logical use, and will allow a different size of technology to be considered. Knowing the factors that influence nanomaterials will make using nanotechnology easier to implement, this is the main focus of this study.

Several studies have been conducted in the literature about vibration and bending of nanorods and the factors which are affecting on nanorods. Ecsedi and Baksa [1] published studies about 
axial vibration of nanorods based on non-local elasticity. Şimşek [2] examined axial vibration of nanorod using non-local strain gradient theory. Yayli [3] investigated axial vibration analysis of a Rayleigh nanorod with deformable boundaries. Aydoğdu [4] published study about axial vibration analysis of carbon nanotubes embedded in an elastic medium. Axial vibration analysis of a tapered nanorod with a differential quadrature method was examined by Danesh et al. [5]. Guo and Yang [6] conducted axial vibration analysis of nanocones based on non-local elasticity theory. Murmu and Pradhan [7] published studies about vibration analysis of nanoplates under uniaxial pre stressed conditions via non-local elasticity. Comparison of various refined nonlocal beam theories for bending, vibration and buckling analysis of beams investigated by Berrabah et al. [8]. Fakher et al [9] published study about bending and free vibration analysis of nanobeams by differential and integral forms of non-local strain gradient with Rayleigh-Ritz method. Akgöz and Civalek [10] indicated buckling analysis of functionally graded microbeams based on the strain gradient theory. Attia and Mahmoud [11] published studies modeling and analysis of nanobeams based on non-local couple-stress elasticity and surface energy theories. Static analysis of nanobeams including surface effects by non-local finite element published by Mahmoud et al. [12]. Norouzzadeh et al. [13] indicated non-linear bending analysis of nanobeams based on non-local strain gradient model using an isogeometric finite element approach. Alshorbagy et al. [14] published study about static analysis of nanobeams using non-local FEM. Coupling effects of non-local and surface energy on vibration analysis of nanobeams investigated by Eltaher et al. [15]. Studies so far show how diverse the vibration and bending analysis is, and then there are many different theories to be revealed. Arefi et al. [16] examined the non-local bending analysis of curved nanobeams reinforced by grapheme nanoplatelets. Lu et al. [17] indicated size-dependent vibration analysis of nanobeams based on the non-local strain gradient theory. A unified non-local formulation for bending, buckling and free vibration analysis of nanobeams published by Nikam and Sayyad [18]. Determination of the appropriate gradient elasticity theory for bending analysis of nanobeams by considering boundary conditions effect investigated by Shokrieh and Zibaei [19]. Beni [20] published study about size-dependent electromechanical bending, buckling and free vibration analysis of functionally graded piezoelectric nanobeams. While this is similar in terms of the subject, it shows us how much factors are effecting on nanomaterials. Ghadiri et al. [21] indicated non-linear forced vibration analysis of nanobeams subjected to moving concentrated load resting on a viscoelastic foundation considering thermal and surface effects. Behera and Chakravery [22] published study about application of differential quadrature method in free vibration analysis of nanobeams based on various non-local theories. In this type of works we frequently use non-local theory and that can be used in many aspects in terms of usage width. Nejad and Hadi [23] investigated non-local analysis of free vibration of bidirectional functionally graded Euler-Bernoulli nanobeams. Ebrahimi and Salari [24] indicated thermal buckling and free vibration analysis of size dependent Timoshenko FG nanobeams in thermal enviroments. In studies like this, main purpose is observing the outside effects on nanomaterials. Lin et al. [25] investigated assessment of first and third order shear deformation beam theories for the buckling and vibration analysis of nanobeams incorporating surface stress effects. Vibration analysis of smart piezoelectrically actuated nanobeams subjected to magneto-electrical field in thermal environment studied by Ebrahimi and Barati [26]. Ansari et al. [27] conducted non-linear vibration analysis of Timoshenko nanobeams based on surface stress elasticity theory. Free vibration analysis of Euler-Bernoulli nanobeam using differential transform method published by Jena and Chakraverty [28]. Forced vibration analysis of functionally graded nanobeams studied by Akbas [29]. Shafiei et al. [30] investigated nonlinear vibration of axially functionally graded non-uniform nanobeams. One reason for the diversification of literature studies is that multiple factors can be addressed in nanorods. Complex modal analysis of transverse free vibrations for axially moving nanobeams 
based on the non-local strain gradient theory published by Wang et al. [31]. Vibration analysis of Euler-Bernoulli nanobeams by using finite element method examined by Eltaher et al. [32]. One other analysis done by Oskouie and Ansari [33] about linear and nonlinear vibrations of fractional viscoelastic Timoshenko nanobeams considering surface energy effects. Zhang et al. [34] investigated analyses of transverse vibrations of axially pre tensioned viscoelastic nanobeams with small size and surface effects. Yang et al. [35] studied nonlinear bending, buckling and vibration of bidirectional functionally graded nanobeams. Another study published by Lv and Liu [36] about nonlinear bending response of functionally graded nanobeams with material uncertainties. As we can see, many researchers have often included factors that affect nanomaterials in their studies. Exact solutions of bending deflections for nanobeams and nanoplates based on non-local elasticity theory published by Yan et al. [37]. Sciarra and Baretta [38] indicated a new non-local bending model for Euler-Bernoulli nanobeams. Finite Element analysis of plates and shells investigated by Civalek [39]. One of the studies about Timoshenko beams was done by Civalek and Kiracioglu in their work called free vibration analysis of Timoshenko beams by DSC method [40]. Mercan et al. examined vibration analysis of FG cylindrical shells with power-law index using discrete singular convolution technique [41].

In this study, it has been examined how nanostructures influence the bending analysis of their changing characteristics with the change in their properties of the particular type. Because boundary conditions can be deformed, it is possible to have a closed solution of this differential equation separately, but it is intended to create general self-value problem using by Modified Couple Stress Theory, and bending analysis of a free-end nanobeam can be found, giving very small values to these elastic boundary conditions. At the same time, the bending analysis of nanobeam and which key parameters effects on nanobeam's bending has been studied in this article. The parameters that have an effect on the bending of the nanobeams has been compared with the calculated values and evaluations has been made on these results.

\section{Bending Analysis of A Nanobeam}

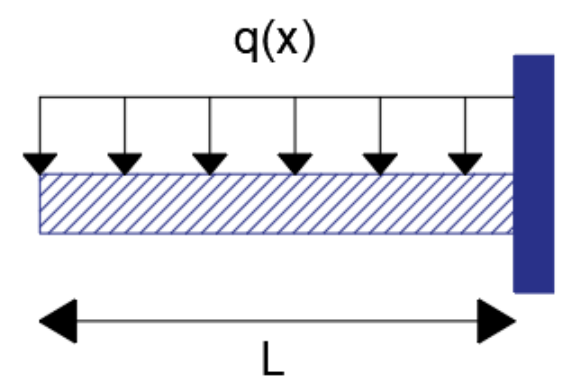

Fig. 1. A cantilever nanobeam which is uniformly loaded

In this section, bending analysis of the uniformly loaded cantilever nanobeam shown in Fig 1 has been made. While performing these analyzes, weighted residual function methods are used. We can define the components for the displacement of Euler-Bernoulli beam as follows:

$$
u=-z \psi(x), v=0, w=w(x)
$$

$\mathrm{u}, \mathrm{v}, \mathrm{w}$ are $\mathrm{u}$ components of the displacement vectors of $\mathrm{x}, \mathrm{y}, \mathrm{z}$ respectively and $\psi$ is the angle of rotation of the center axis. 


$$
\psi \approx \frac{d w(x)}{d x}
$$

Using Eqs. (1,2):

$$
\varepsilon_{x x}=\frac{d u}{d x}=-z \frac{d^{2} w(x)}{d x^{2}}, \varepsilon_{y y}=\varepsilon_{z z}=\varepsilon_{x y}=\varepsilon_{y z}=\varepsilon_{z x}=0
$$

equaliton is obtained [47]. From the Eqs. $(1,2)$ given above:

$$
\theta_{y}=\frac{d w(x)}{d x}, \theta_{x}=\theta_{z}=0
$$

calculation is acquired [47]. From the Eq. (4) given above, the following relation is received:

$$
\chi_{x y}=-\frac{1}{2} \frac{d^{2} w(x)}{d x^{2}}, \chi_{x x}=\chi_{y y}=\chi_{z z}=\chi_{y z}=\chi_{z x}=0
$$

Using the equations obtained above:

$$
\begin{gathered}
\sigma_{x x}=\frac{E(1-v)}{(1+v)(1-2 v)}\left(-z \frac{d^{2} w(x)}{d x^{2}}\right), \sigma_{y y}=\sigma_{z z}=\frac{E v}{(1+v)(1-2 v)}\left(-z \frac{d^{2} w(x)}{d x^{2}}\right) \\
\sigma_{x y}=\sigma_{y z}=\sigma_{z x}=0
\end{gathered}
$$

this equation is acquired.

In the equation obtained above, E represents Young's modulus and $v$ represents the Poisson ratio. E and $v$ are related to the Lame constants $\lambda$ and $\mu$.

$$
\begin{gathered}
\lambda=\frac{E v}{(1+v)(1-2 v)} \\
\mu=\frac{E}{2(1+v)}
\end{gathered}
$$

As defined in Eq. (8), $\mu$ is known as the material constant. This expression is also known as shear modulus $(\mathrm{G})$. For a beam with a high aspect ratio, the Poisson effect can be neglected to more easily express the simple beam theory formulation. And the equation created in this way is as follows:

$$
\sigma_{x x}=-E z \frac{d^{2} w(x)}{d x^{2}}, \sigma_{i j}=0
$$

If the equation is replaced in the relevant relations:

$$
m_{x y}=\mu \mathrm{I}_{2}^{2} \frac{d^{2} w(x)}{d x^{2}}, m_{x x}=m_{y y}=m_{z z}=m_{y z}=m_{z x}=0
$$

When this calculation is acquired [47]. Using Eqs. $(3,6,9,10)$ the following equation is obtained. 


$$
U=-\frac{1}{2} \int_{x=0}^{L} M_{x} \frac{d^{2} w(x)}{d x^{2}} d x-\frac{1}{2} \int_{x=0}^{L} Y_{x y} \frac{d^{2} w(x)}{d x^{2}} d x
$$

The closed equation created after the calculations is as follows [47]:

$$
w(x)=\frac{1}{\left(E I+\mu A I_{2}^{2}\right)}\left[-\frac{q x^{4}}{72}+c_{1} x+c_{2}\right]
$$

\section{Weighted Residual Methods}

\subsection{Sub-domain method}

The application of this method is achieved by taking the residual function of the integral over the selected areas as zero. The residual function in modified couple stress theory is given below:

$$
R=\left(E I+\mu * A * \mathfrak{I}_{2}^{2}\right) * \frac{\partial^{2} W}{\partial x^{2}}+\frac{q * x^{2}}{2}=0
$$

Following approximation function is used in this study [14]:

$$
\begin{gathered}
W=a_{1} *(x-L)^{2}+a_{2} *(x-L)^{3}+a_{3} *(x-L)^{4} \\
\mathrm{R}=\left(\mathrm{EI}+\mu * \mathrm{~A} * \mathrm{I}_{2}^{2}\right) *\left(2 * \mathrm{a}_{1}+6 * \mathrm{a}_{2} *(\mathrm{x}-\mathrm{L})+12 * \mathrm{a}_{3} *(\mathrm{x}-\mathrm{L})^{2}\right)+\frac{\mathrm{q}^{2} \mathrm{x}^{2}}{2} \\
\int_{0}^{\frac{L}{3}}\left(\left(E I+\mu * A * \mathrm{I}_{2}^{2}\right) *\left(2 * a_{1}+6 * a_{2} *(x-L)+12 * a_{3} *(x-L)^{2}\right)+\frac{q * x^{2}}{2}\right) d x=0 \\
\int_{\frac{L}{3}}^{\frac{2 L}{3}}\left(\left(E I+\mu * A * \mathrm{I}_{2}^{2}\right) *\left(2 * a_{1}+6 * a_{2} *(x-L)+12 * a_{3} *(x-L)^{2}\right)+\frac{q * x^{2}}{2}\right) d x=0 \\
\int_{\frac{2 L}{3}}^{L}\left(\left(E I+\mu * A * \mathrm{I}_{2}^{2}\right) *\left(2 * a_{1}+6 * a_{2} *(x-L)+12 * a_{3} *(x-L)^{2}\right)+\frac{q * x^{2}}{2}\right) d x=0
\end{gathered}
$$

After the integrals are taken, following equations are obtained:

$$
\begin{aligned}
& \frac{1}{162} L\left(L^{2} q+6\left(\mathrm{EI}+\mu * A * I_{2}^{2}\right)\left(18 a_{1}+L\left(-45 a_{2}+76 \mathrm{La}_{3}\right)\right)\right)=0 \\
& \frac{1}{162} L\left(7 L^{2} q+6\left(E I+\mu * A * \mathfrak{I}_{2}^{2}\right)\left(18 a_{1}+L\left(-27 a_{2}+28 L a_{3}\right)\right)\right)=0 \\
& \frac{1}{162} L\left(19 L^{2} q+6\left(E I+\mu * A * \mathfrak{I}_{2}^{2}\right)\left(18 a_{1}+L\left(-9 a_{2}+4 L a_{3}\right)\right)\right)=0
\end{aligned}
$$

Once this equation system is solved, the unknown coefficients are found:

$$
\begin{aligned}
& a_{1}=-\frac{L^{2} q}{4\left(E I+\mu * A * I_{2}^{2}\right)} \\
& a_{2}=-\frac{L q}{6\left(E I+\mu * A * I_{2}^{2}\right)}
\end{aligned}
$$




$$
a_{3}=-\frac{q}{24\left(E I+\mu * A * I_{2}^{2}\right)}
$$

These coefficients are written in the calculation and the $W$ function becomes as in Eq. (25):

$$
\begin{gathered}
W=-\frac{L^{2} q}{4\left(E I+\mu * A * I_{2}^{2}\right)} *(x-L)^{2}-\frac{L q}{6\left(E I+\mu * A * I_{2}^{2}\right)} *(x-L)^{3}-\frac{q}{24\left(E I+\mu * A * I_{2}^{2}\right)} \\
*(x-L)^{4}
\end{gathered}
$$

\subsection{Galerkin method}

When solving with the Galerkin method, three boundary condition relations are chosen for three unknown coefficients:

$$
\begin{aligned}
\int_{0}^{L} R * W_{i} d x & =0 i=0,1,2 \ldots N \\
W_{1} & =(x-L) \\
W_{2} & =(x-L)^{2} \\
W_{3} & =(x-L)^{3}
\end{aligned}
$$

Using the approximation functions given above, the following equations are obtained when substituted sequentially in Eq. (26):

$$
\begin{gathered}
\int_{0}^{L}\left(\left(E I+\mu * A * \mathrm{I}_{2}^{2}\right) *\left(2 * a_{1}+6 * a_{2} *(x-L)+12 * a_{3} *(x-L)^{2}\right)+\frac{q * x^{2}}{2}\right) * \\
(x-L)^{2} d x=0 \\
\int_{0}^{L}\left(\left(E I+\mu * A * I_{2}^{2}\right) *\left(2 * a_{1}+6 * a_{2} *(x-L)+12 * a_{3} *(x-L)^{2}\right)+\frac{q * x^{2}}{2}\right) * \\
(x-L)^{2} d x=0 \\
\int_{0}^{L}\left(\left(\mathrm{EI}+\mu * \mathrm{~A} * \mathrm{I}_{2}^{2}\right) *\left(2 * a_{1}+6 * a_{2} *(x-L)+12 * a_{3} *(x-L)^{2}\right)+\frac{q * x^{2}}{2}\right) * \\
(x-L)^{3} d x=0
\end{gathered}
$$

The integrals given above are calculated, the following system of calculations is formed:

$$
\begin{array}{r}
-\frac{L^{4} q}{24}-L^{2}\left(E I+\mu * A * I_{2}^{2}\right)\left(a_{1}+L\left(-2 a_{2}+3 L a_{3}\right)\right)=0 \\
\frac{1}{60} L\left(L^{4} q+6 L^{2}\left(E I+\mu * A * I_{2}^{2}\right)\left(20 a_{1}+9 L\left(-5 a_{2}+8 L a_{3}\right)\right)\right)=0
\end{array}
$$




$$
\frac{1}{120} L^{4}\left(-L^{2} q-36\left(E I+\mu * A * I_{2}^{2}\right)\left(5 a_{1}+4 L\left(-3 a_{2}+5 L a_{3}\right)\right)\right)=0
$$

The equation system is solved, the unknowns $a_{1}, a_{2}, a_{3}$ are found as follows:

$$
\begin{aligned}
& a_{1}=-\frac{L^{2} q}{4\left(E I+\mu * A * I_{2}^{2}\right)} \\
& a_{2}=-\frac{L q}{6\left(E I+\mu * A * I_{2}^{2}\right)} \\
& a_{3}=-\frac{q}{24\left(E I+\mu * A * I_{2}^{2}\right)}
\end{aligned}
$$

When the found unknown coefficients are replaced in the $W$ function, the equation to be formed is as:

$$
\begin{gathered}
W=-\frac{L^{2} q}{4\left(E I+\mu * A * I_{2}^{2}\right)} *(x-L)^{2}-\frac{L q}{6\left(E I+\mu * A * I_{2}^{2}\right)} *(x-L)^{3}-\frac{q}{24\left(E I+\mu * A * I_{2}^{2}\right)} \\
*(x-L)^{4}
\end{gathered}
$$

\section{Analysis and Numerical Results}

The values obtained as a result of the three-parameter solution method using the weighted residual methods are given in Table 1. The graphic in Fig.2 was created using Table 1. The values acquired by solving the two-parameter solution method by using the weighted residual method are given in Table 2. And by using Table 2, the results received by using the twoparameter solution method and the weighted residual methods are compared in Fig.3. While drawing the graphs in Figures 2 and 3, the numerical values in Tables 1 and 2 were used.

Table 1. Comparison of the calculated values with the weighted residual methods of the threeparameter solution that we used in the study

\begin{tabular}{lcccccc}
\hline & $\mathbf{x}=\mathbf{0}$ & $\mathbf{x}=\mathbf{2}$ & $\mathbf{x}=\mathbf{4}$ & $\mathbf{x}=\mathbf{6}$ & $\mathbf{x}=\mathbf{8}$ & $\mathbf{x}=\mathbf{1 0}$ \\
\hline $\mathrm{W}_{\text {Subdomain }}$ & $-1246,26$ & $-914,59$ & $-592,223$ & $-303,091$ & $-87,0721$ & 0 \\
$\mathrm{~W}_{\text {Galerkin }}$ & $-1246,26$ & $-914,59$ & $-592,223$ & $-303,091$ & $-87,0721$ & 0 \\
$\mathrm{~W}_{\text {General }}[47]$ & $-1246,26$ & $-914,59$ & $-592,223$ & $-303,091$ & $-87,0721$ & 0 \\
\hline
\end{tabular}

The displacement values of an axially loaded nanobeams are given by using different weighted residual methods given in the table. It has been shown that the values calculated by applying different solution methods and also the calculated implicit solution value are the same. 


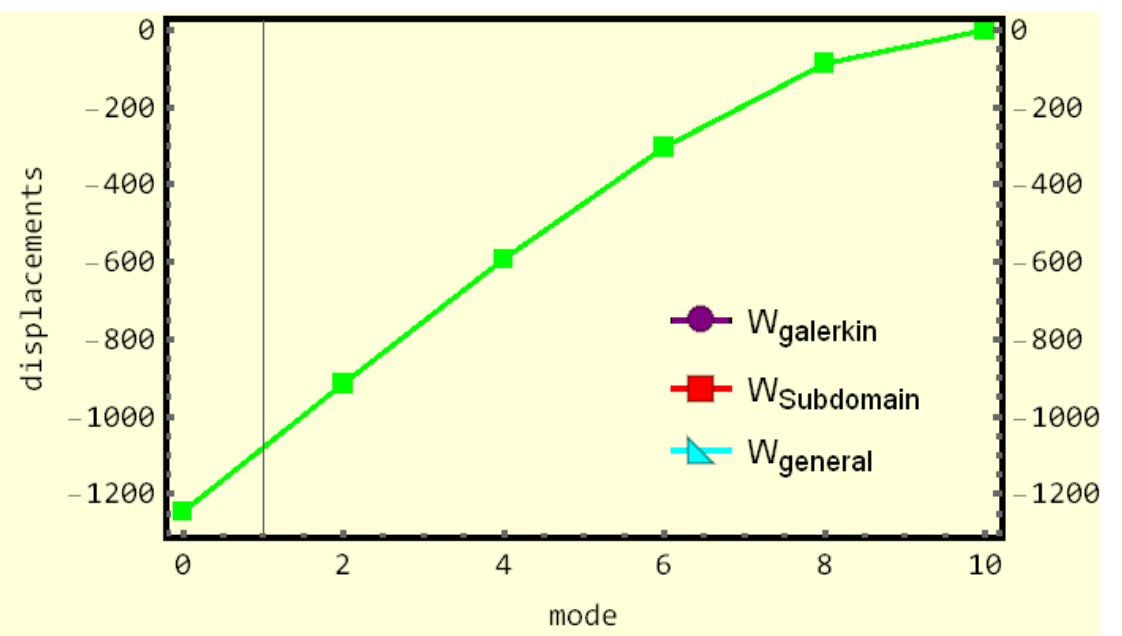

Fig. 2. Comparison of the three-parameter solution with weighted residual methods

Table 2. Comparison of the calculated values of the two-parameter solution with the weighted residual methods

\begin{tabular}{lcccccc}
\hline & $\mathbf{x}=\mathbf{0}$ & $\mathbf{x}=\mathbf{2}$ & $\mathbf{x}=\mathbf{4}$ & $\mathbf{x}=\mathbf{6}$ & $\mathbf{x}=\mathbf{8}$ & $\mathbf{x}=\mathbf{1 0}$ \\
\hline $\mathrm{W}_{\text {Subdomain }}$ & $-1246,26$ & $-914,59$ & $-592,223$ & $-303,091$ & $-87,0721$ & 0 \\
$\mathrm{~W}_{\text {Galerkin }}$ & $-1080,09$ & $-776,33$ & $-484,546$ & $-236,623$ & $-64,473$ & 0 \\
$\mathrm{~W}_{\text {General }}[47]$ & $-1246,26$ & $-914,59$ & $-592,223$ & $-303,091$ & $-87,0721$ & 0 \\
\hline
\end{tabular}

The displacement values of an axially loaded nanobeams are given by using different weighted residual methods given in Table 2 . In the previous study, it has been observed that all values are the same in the three-parameter solution calculation, but not all values are the same in the two-parameter solution.

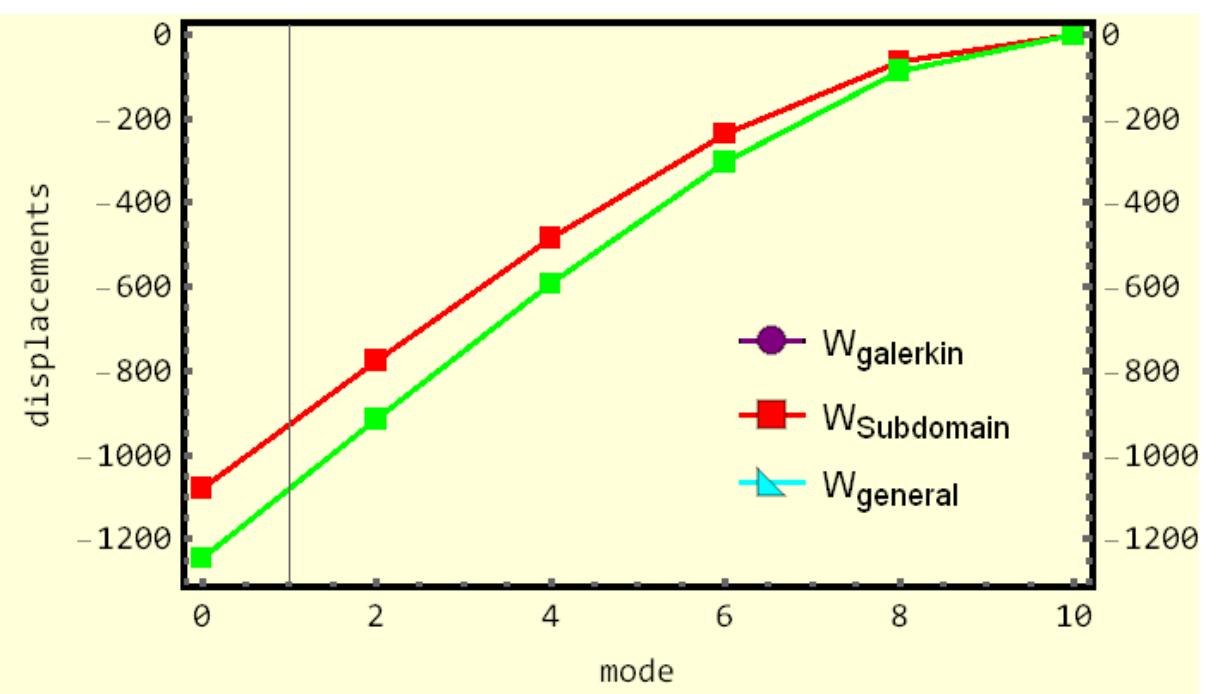

Fig. 3. Comparison of two parameter solution with weighted residual function solution methods 


\section{Conclusion}

Within the scope of this study, the bending analysis of nanobeams has been analyzed using different weighted residual methods. The results of the three-parameter solution method gave the same result as the implicit equation to all weighted residual methods applied. In the twoparameter method, the closed equation gave the same result with all methods except for the Galerkin method. In this direction, the values of the two-parameter solution method and the three-parameter solution method are presented in the article with tables and graphs after the solutions of the bending analysis are completed.

\section{References}

[1] Ecsedi, I., Baksa, A., Free axial vibration of nanorods with elastic medium interaction based on nonlocal elasticity, Mechanics Research Communications, 86, 2017.

[2] Simsek, M., Nonlocal effects in the free longitudinal vibration of axially functionally graded tapered nanorods, Computational Materials Science, 61, 257-265, 2012.

[3] Yaylı, M.Ö., Buckling Analysis of a Rotationally Restrained Single Walled Carbon Nanotube Embedded in an Elastic Medium Using Nonlocal Elasticity, International Journal of Engineering \& Applied Sciences, 8(2), 40-50, 2016.

[4] Aydogdu, M., A general nonlocal beam theory: its application to nanobeam bending, buckling and vibration, Physica E: low-dimensional systems and nanostructures, 41(9), 1651-1655, 2009.

[5] Danesh, M., Farajpour, A., Mohammadi, M., Axial vibration analysis of a tapered nanorod based on nonlocal elasticity theory and differential quadrature method, Mechanics Research Communications, 39, 23-27, 2012.

[6] Guo, S.Q, Yang, S.P., Axial vibration analysis of nanocones based on nonlocal elasticity theory, Acta Mech. Sin., 28(3), 801-807, 2012.

[7] Murmu, T., Pradhan, S.C., Vibration analysis of nanoplates under uniaxial prestressed conditions via nonlocal elasticity, J. Appl. Phys., 106, 104301, 2009.

[8] Berrabah, H.M., Tounsi, A., Semmah, A., Adda Bedia, E.A., Structural Engineering and Mechanics, 48(3), 351-365, 2013.

[9] Faker, M., Bending and free vibration analysis of nanobeams by differential and integral forms of nonlocal strain gradient with Rayleigh-Ritz method, Mater. Res. Express, 20531591, 2017.

[10] Akgöz, B., Civalek, Ö., Buckling analysis of functionally graded microbeams based on the strain gradient theory, Acta Mechanica, 224, 2185-201, 2013.

[11] Attia, M.A., Mahmoud, F.F., Modeling and Analysis of Nanobeams Based on Nonlocal Couple-Stress Elasticity and Surface Energy Theories, International Journal of Mechanical Sciences, 105, 126-134, 2016. 
[12] Mahmoud, F.F., Eltaher, M.A., Alshorbagy, A.E, Meletis, E.I., Static analysis of nanobeams including surface effects by nonlocal finite element, Journal of Mechanical Science and Technology, 26(11), 3555-3563, 2012.

[13] Norouzzadeh, A., Ansari, R., Rouhi, H., Nonlinear Bending Analysis of Nanobeams Based on the Nonlocal Strain Gradient Model Using an Isogeometric Finite Element Approach, Iran J Sci Technol Trans Civ Eng, 43(1), 533-547, 2018.

[14] Amal, A.E., Eltaher, M.A., Mahmoud, F.F., Static analysis of nanobeams using nonlocal FEM, Journal of Mechanical Science and Technology, 27(7), 2035-2041, 2013.

[15] Eltaher, M.A., Mahmoud, F.F., Assie, A.E., Meletis, E.I., Coupling effects of nonlocal and surface energy on vibration analysis of nanobeams, Applied Mathematics and Computation 224, 760-774, 2013.

[16] Arefi, M., Mohammad-Rezaei Bidgoli, E., Dimitri, R., Bacciocchi, M., Tornabene, F., Nonlocal bending analysis of curved nanobeams reinforced by graphene nanoplatelets, Composites Part B, 166, 1-12, 2018.

[17] Lu, L., Guo, X., Zhao, J., Size-dependent vibration analysis of nanobeams based on the nonlocal strain gradient theory, International Journal of Engineering Science, 116, 12-24, 2017.

[18] Nikam, R.D., Sayyad, A.S., A unified nonlocal formulation for bending buckling and free vibration analysis of nanobeams, Mechanics of Advanced Materials and Structures, 27, 807-815, 2018.

[19] Shokrieh, M., Zibaei, I., Determination of the Appropriate Gradient Elasticity Theory for Bending Analysis of Nano-beams by Considering Boundary Conditions Effect, Latin American Journal of Solids and Structures, 12, 2208-2230, 2014.

[20] Beni, Y., Size-dependent electromechanical bending, buckling, and free vibration analysis of functionally graded piezoelectric nanobeams, Journal of Intelligent Material Systems and Structures, 27(16), 2199-2215, 2016.

[21] Ghadiri, M., Rajabpour, A., Akbarshahi, A., Non-linear forced vibration analysis of nanobeams subjected to moving concentrated load resting on a viscoelastic foundation considering thermal and surface effects, Applied Mathematical Modelling, 50, 676-694, 2017.

[22] Behera, L., Chakraverty, S., Application of Differential Quadrature method in free vibration analysis of nanobeams based on various nonlocal theories, Computers and Mathematics with Applications, 69(12), 1444-1462, 2015.

[23] Hadi, A., Nejad M., Hosseini, M., Vibrations of three-dimensionally graded nanobeams, International Journal of Engineering Science, 128, 12-23, 2018. 
[24] Ebrahimi, F., Salari, E., Thermal buckling and free vibration analysis of size dependent Timoshenko FG nanobeams in thermal environments, Composite Structures, 128, 363-380, 2015.

[25] Lin, S.C., Hsiao, K.M., Vibration analysis of a rotating Timoshenko beam, Journal of Sound and Vibration, 240(2), 303-322, 2001.

[26] Ebrahimi, F., Barati, M., Vibration analysis of smart piezoelectrically actuated nanobeams subjected to magneto-electrical field in thermal environment, Journal of Vibration and Control, 24(3), 549-564, 2016.

[27] Ansari, R., Mohammadi, V., Faghih Shojaei, M., Gholami, R., Rouhi, H., Nonlinear vibration analysis of Timoshenko nanobeams based onsurface stress elasticity theory, European Journal of Mechanics, 45, 143-152, 2014.

[28] Jena, S., Chakraverty, S., Free vibration analysis of Euler-Bernoulli Nano beam using differential transform method, International Journal of Computational Materials Science and Engineering, 7(3), 2018.

[29] Akbas, S., Forced vibration analysis of functionallygraded nanobeams, International Journal of Applied Mechanics, 7(4), 736-743, 2017.

[30] Shafiei, N., Kazemi, M., Safi, M., Ghadiri, M., Nonlinear vibration of axially functionally graded non-uniform nanobeams, International Journal of Engineering Science, 106, 7794, 2016.

[31] Wang, J., Shen, H., Zhang, B., Liu, J., Zhang, Y., Complex modal analysis of transverse free vibrations for axially moving nanobeams based on the nonlocal strain gradient theory, Physica E: Low-dimensional Systems and Nanostructures, 14(1),119-137, 2018.

[32] Eltaher, M.A., Alshorbagy, A. E., Mahmoud, F.F., Vibration analysis of Euler-Bernoulli nanobeams by using finite element method, Applied Mathematical Modelling, 37, 47874797, 2013.

[33] Faraji Oskouie, M., Ansari, R., Linear and nonlinear vibrations of fractional viscoelastic Timoshenko nanobeams considering surface energy effects, Applied Mathematical Modelling, 43(3), 337-350, 2016.

[34] Zhang, R., Pang, M., Fan, M., Analyses of transverse vibrations of axially pretensioned viscoelastic nanobeams with small size and surface effects, Physics Letters A, 380(29-30), 2294-2299, 2016.

[35] Yang, T., Tang, Y., Li, Q., Yang, X-D., Nonlinear bending, buckling and vibration of bidirectional functionally graded nanobeams, Composite Structures, 156, 319-331, 2018.

[36] Zheng, L., Liu, H., Nonlinear bending response of functionally graded nanobeams with material uncertainties, International Journal of Mechanical Sciences, 12, 134, 2017. 
[37] Yan, J.W., Tong, L.H., Li, C., Zhu, Y., Wang, Z.W., Exact Solutions of Bending Deflections for Nano-beams and Nano- plates Based on Nonlocal Elasticity Theory, Composite Structures, 125, 304-313, 2015.

[38] Barretta, R., Sciarra, R., A new nonlocal bending model for Euler-Bernoulli nanobeams, Mechanics Research Communications, 62, 25-30, 2014.

[39] Civalek, O., Finite Element analysis of plates and shells, Master thesis, Elazı $\breve{g}$, Firat University, 1998. (in Turkish)

[40] Civalek, O., Kiracioglu, O., Free vibration analysis of Timoshenko beams by DSC method, International Journal for Numerical Methods in Biomedical Engineering, 26(12), 1890-1898, 2010.

[41] Mercan, K., Demir, Ç., Civalek, O., Vibration analysis of FG cylindrical shells with powerlaw index using discrete singular convolution technique, Curved and Layered Structures, 3(1), 82-90, 2016.

[42] Demir, C., Civalek, O., On the analysis of microbeams, International Journal of Engineering Science, 121, 14-33, 2017.

[43] Jalaei, M., Civalek, O., On dynamic instability of magnetically embedded viscoelastic porous FG nanobeam, International Journal of Engineering Science, 143, 14-32, 2019.

[44] Civalek, O., Dastjerdi, S., Akbaş, S.D., Akgöz, B., Vibration Analysis of Carbon Nanotube-Reinforced Composite Microbeams. Mathematical Methods in the Applied Sciences, 11(3), 571, 2020.

[45] Civalek, Ö., Avcar, M., Free vibration and buckling analyses of CNT reinforced laminated non-rectangular plates by discrete singular convolution method. Engineering with Computers, 1-33, 2020.

[46] Hadji, L., Avcar, M., Nonlocal free vibration analysis of porous FG nanobeams using hyperbolic shear deformation beam theory, Advances in Nano Research, 10(3), 281-293, 2021.

[47] Akgöz, B., Linear and nonlinear analyses of micro and nano structures based on higherorder elasticity theories, Doktora Tezi, Akdeniz Üniversitesi, 2010.

[48] Yayl1, M.Ö, Çerçevik, E.A., Axial vibration analysis of cracked nanorods with arbitrary boundary conditions, Journal of Vibroengineering, 17(6), 2907-2921, 2015.

[49] Uzun, B., Yayl1, M.Ö., Deliktaş, B., Free vibration of FG nanobeam using a finite-element method, Micro \& Nano Letters, 15(1), 35-40, 2020.

[50] Yayli, M.Ö., Buckling Analysis of a Rotationally Restrained Single Walled Carbon Nanotube, Acta Physica Polonica A, 127, 3, 678-683, 2015.

[51] Yayli, M.Ö., Stability analysis of a gradient elasticbeam using finite element method, International Journal of the Physical Sciences, 6(12), 2844-2851, 2011. 
[52] Yayli, M.Ö., Free Vibration Behavior of a Gradient Elastic Beam with Varying Cross Section, Shock and Vibration, vol. 2014, Article ID 801696, 11 pages, 2014.

[53] Yayli, M.Ö., Torsional vibration analysis of nanorods with elastic torsional restraints using non-local elasticity theory, Micro \& Nano Letters, 13(5), 595-599, 2018.

[54] Yayli, M.Ö., A compact analytical method for vibration of micro-sized beams with different boundary conditions, Mechanics of Advanced Materials and Structures, 24(6), 496-508, 2017.

[55] Yayli, M.Ö., Torsion of nonlocal bars with equilateral triangle cross sections, Journal of Computational and Theoretical Nanoscience, 10(2), 376-379, 2013.

[56] Yayl1, M.Ö., Buckling analysis of a rotationally restrained single walled carbon nanotube embedded in an elastic medium using nonlocal elasticity, International Journal of Engineering and Applied Sciences, 8(2), 40-50, 2016.

[57] Yayli, M.Ö., Weak formulation of finite element method for nonlocal beams using additional boundary conditions, Journal of Computational and Theoretical Nanoscience, 8(11), 2173-2180, 2011.

[58] Yayl1, M. Ö., An analytical solution for free vibrations of a cantilever nanobeam with a spring mass system, International Journal of Engineering and Applied Sciences, 7(4), 10$18,2016$.

[59] Kadığlu, H. \& Yaylı, M. Ö., Buckling analysis of non-local Timoshenko beams by using Fourier series, International Journal of Engineering and Applied Sciences, 9(4), 89-99, 2017.

[60] Uzun, B, \& Yaylı, M. Ö., Nonlocal vibration analysis of Ti-6Al-4V/ZrO 2 functionally graded nanobeam on elastic matrix, Arabian Journal of Geosciences, 13(4), 1-10, 2020. 